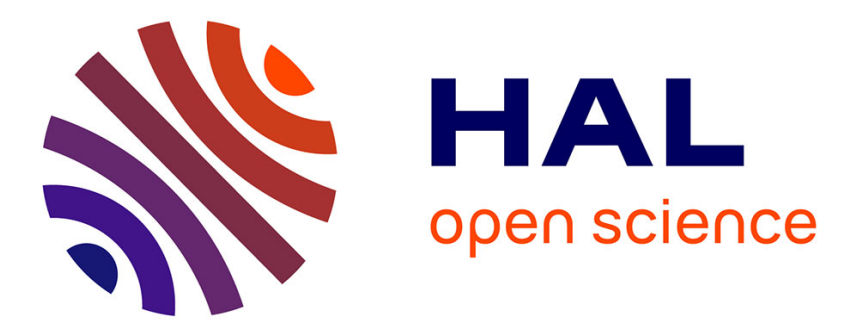

\title{
Challenges in Knowledge Intensive Process Management
} Fabrice Boissier, Irina Rychkova, Bénédicte Le Grand

\section{To cite this version:}

Fabrice Boissier, Irina Rychkova, Bénédicte Le Grand. Challenges in Knowledge Intensive Process Management. 2019 IEEE 23rd International Enterprise Distributed Object Computing Workshop (EDOCW), Oct 2019, Paris, France. pp.65-74, 10.1109/EDOCW.2019.00023 . hal-02561485

\section{HAL Id: hal-02561485 \\ https://hal.science/hal-02561485}

Submitted on 4 May 2020

HAL is a multi-disciplinary open access archive for the deposit and dissemination of scientific research documents, whether they are published or not. The documents may come from teaching and research institutions in France or abroad, or from public or private research centers.
L'archive ouverte pluridisciplinaire HAL, est destinée au dépôt et à la diffusion de documents scientifiques de niveau recherche, publiés ou non, émanant des établissements d'enseignement et de recherche français ou étrangers, des laboratoires publics ou privés. 


\title{
Challenges in Knowledge Intensive Process Management
}

\author{
Fabrice Boissier \\ Irina Rychkova \\ Bénédicte Le Grand \\ Centre de Recherche en Informatique, Centre de Recherche en Informatique, Centre de Recherche en Informatique, \\ Université Paris 1 - Panthéon Sorbonne, Université Paris 1 - Panthéon Sorbonne, Université Paris 1 - Panthéon Sorbonne, \\ 90 rue de Tolbiac, 75013, Paris, France 90 rue de Tolbiac, 75013, Paris, France 90 rue de Tolbiac, 75013, Paris, France \\ fabrice.boissier@univ-paris1.fr_ｉrina.rychkova@univ-paris1.fr_benedicte.le-grand@univ-paris1.fr
}

\begin{abstract}
Processes where knowledge is a key characteristic are called knowledge intensive processes (KIP). A successful KIP has to adapt for the situation and to treat each customer's request as unique rather than to follow some predefined sequence of actions. The discipline of Business Process Management (BPM) defines solutions for modeling, development, analysis and improvement of processes with a predefined flow of activities. From the traditional, activity-centered point of view, KIP are challenging to automate, to control and to test for compliance.

In this article we present the overview of recent works that address the challenges and explore different ideas, including extension of BPM, theoretical foundations for KIP management and execution support for KIP. We also outline some research perspectives in KIP management and discuss one particular idea that exploits the data-centered point of view on KIP.

Keywords-Knowledge Intensive Process, Knowledge Intensive Business Process, Adaptive Case Management, Business Process Management.
\end{abstract}

\section{INTRODUCTION}

The term process comes from Latin and refers to a performed action or something that has been done to achieve a specific result [1]. No surprise that since Adam Smith and Frederick Taylor the de facto point of view on business processes was activity-centered, where business process are represented with a set of interrelated and ordered activities or tasks.

Today, in the era of Data, a new point of view on the business processes has emerged. For many processes, success is not anymore in their predictability and efficiency, but in their capacity to adapt for the situation and to treat each customer's request as unique. Such processes require creativity and collaboration. Their execution depends on the data collected for the current process instance or case and on the knowledge of an expert handling the case, rather than on the à priori model. These are knowledge-intensive processes (KIP).

We execute KIP to achieve a specific result, however the way we achieve it is not any more a universal sequence of actions that can be designed, validated and tested before execution. Thus, from the traditional, activity-centered point of view, KIP are challenging to automate, to control their execution and improve them.

Various communities have grown with the objective to better study KIP and to share practices. ACM (adaptive case management) [2] is the community of practitioners promoting the methods and tools for adaptive case management as opposed to business process management. The WfMC Global Awards (Workflow Management Coalition) for Case Management recognizes and focuses upon successful use cases within Adaptive Case Management. CMMN (Case Management Modeling and Notation) [3][4] is the OMG case modeling standard. It also gathers a community of companies around the representation and support of cases, including IBM, Oracle, TIBCO, SAP and others. AdaptiveCM (International Workshop on Adaptive Case Management) and DEC2H (DECision and Hybrid approaches to processes) are annual workshops where researchers and practitioners publish and discuss about KIP and other non-workflow approaches for process management. Problems around automation, collaboration, flexibility, compliance, and structure have been raised. Other communities work on new approaches, like Speech-Act [5] or AI4BPM (Artificial Intelligence for Business Process Management).

Business Process Management (BPM) discipline emerged in order to better manage and optimize processes [6]. BPM explores an activity-centered view on the processes, which is based on the assumption that any business process can be modeled with a (possibly large) sequence(s) of activities. Process activities according to BPM are interrelated, ordered in time, and known à priori. The process model can be tested and validated; the resulting process will then follow (instantiate) this model at each execution. This activity-centered view provides control over processes and ensures compliance "by design". The importance and complexity of KIP were exposed by the progress of BPM within companies: KIP scenarios can be hardly predefined and compliance for KIP can be ensured only at "run-time". This makes application of traditional BPM practices for KIP difficult if at all possible and limits the automation of KIP.

Strategy models, deciding important tasks to plan and which key processes should be optimized, rely on organization's goals [7]. In goals modeling, businesses' stakeholders are interested in the who, what, and why questions [8]. These activities share common practices and challenges with KIP: goals are the most important concepts to keep in mind; information processing, collaboration, and decision making are regular steps; assure compliance or constraint while executing a process is important. 
In this work, we study and analyze the challenges and practices in KIP management reported in the research literature. The objective is to highlight recurrent topics in the KIP literature, preferably on recent works, and characteristics that are still few or unsupported.

In spite the reported advancements, KIP management remains challenging and new research questions are yet to be solved. We outline some perspectives in KIP research. In particular, we discuss an approach to structural and semantic analysis of KIP scenarios. This approach aims to provide the compliance support for KIP by suggesting process fragments fitting the execution context. The discussed solution contributes to the third group of solutions outlined above (execution support). It is based on classification techniques used in data science (for structural analysis), on ontologies (for semantic), and on association rules (for behavior).

The paper is organized as follows. Section II presents the BPM context and defines KIP. Section III presents and discusses the three groups of solutions we identify in the literature: BPM extensions, theoretical foundations for KIP, execution support solutions for KIP. Section IV outlines the perspectives in KIP research. Section V presents our conclusions.

\section{Business Process Management, Knowledge INTENSIVE PROCESSES, AND CASES}

\section{A. Business Process Management}

The literature defines a business process as a set of interrelated activities that are performed in coordination in an organizational and technical environment, in order to achieve a business goal [9][10].

Business process management is a discipline that includes concepts, methods and techniques to support the design, administration, configuration, enactment, and analysis of business process [10]. BPM seeks to manage and optimize business processes in order to deliver the best outputs (product, service, ...) $[6]$.

BPM recognises the 6 phases of the process lifecycle [6]: process identification, process discovery/process modeling, process analysis, process redesign/process improvement, process implementation, process monitoring and controlling.

BPM uses the activity-centered point of view on the processes, where activities define the states of process entities. BPMN [11] (Business Process Model and Notation) and EPC [12] (Event-driven Process Chain) are examples of modeling languages, which are widely used by academics and practitioners. In these modeling languages, the activities and events are defined and explicitly ordered forming workflows that will be executed.

Other modeling languages, less restrictive, also exist under the declarative paradigm. These languages propose constraints as basis for modeling [13].

To support management and optimization of business processes, multiple IT solutions were developed. Process Aware Information Systems (PAIS) are systems that "support operational business processes by combining advances in in- formation technology with recent insights from management science" [13]. Workflow Management Systems (WfMS), an example of a PAIS, are systems for process modeling and/or automation. A process represented by a workflow model is the core of a WfMS [6]. Business Process Management Systems (BPMS) are the successors of WfMS. They support different features for process monitoring and use business intelligence for process improvement [6].

\section{B. Knowledge Intensive Processes}

Process automation and optimization with PAIS, exposed a specific type of processes, difficult to manage within the established BPM paradigm. From industrial processes to current service processes, automation encountered the "knowledgeintensive economy" [14]. Since a few decades, knowledge is considered as one of the most important competitive advantage for companies [15][16][17]. The knowledge management (KM) discipline studies "how knowledge is created, developed, retained and applied in the workplace and how it enables organizational learning and innovation" [18].

In the context of business processes, some specific human activities couldn't easily be modeled with regular process modeling languages, and therefore, were left as dedicated activities or sub-processes [19].

Processes where knowledge is a key characteristics are called knowledge intensive processes (KIP). Compared to manual tasks defined in BPMN 2.0 [11], where a process participant accomplishes a manual task, knowledge-intensive activities or tasks are characterized by their intensity in knowledge and by the requirements to use and transform this knowledge within processes. KIP emphasizes the importance of the experience or tacit knowledge of process participants also called knowledge workers. The role of knowledge workers is to accomplish specific activities that are characterized by complexity or require creativity.

KM and KIP open a fairly new research field where many definitions and characteristics co-exist. The following characteristics of KIP are defined in [14]:

- KIP embed a lot of knowledge: the tasks are complex; the decision making requires a lot of information to be processed simultaneously.

- KIP require a lot of human collaboration to execute the process.

- KIP have a high degree of uncertainty: the activities might not follow the expected order, and activities that were not initially planned might appear. This makes the structure of KIP very loose.

- KIP require creativity: original ideas and improvisation.

Traditional business processes may have some of these characteristics too. However, in the case of KIP, all of them are typically present, making a process very difficult to model and manage within the BPM paradigm.

Concerning uncertainty, a distinction must be done between the ordering of activities, and the unpredictable activities. Unpredictable activities depends on the context of execution and require improvisation based on tacit knowledge. 
For example, in some unique situations, it is expected that knowledge workers improvise or try new actions based on their assumptions (tacit knowledge).

Several terms are used for KIP in the literature: knowledge intensive business process (KIBP) [20][14] or artful process [21][22], which highlights the fact that an art is required to execute them, and it would be extremely difficult to transpose it within an IT system. In this article, we will use the term KIP to denote all of those types of processes.

\section{Adaptive Case Management}

As the KIP field grows and exposes limitations of BPM support, some attempts to support KIP within IS evolved around case paradigm [23]. A community of practitioners, known as ACM, worked specifically on this point of view [2] and in what extent cases can handle KIP challenges [24][25].

Following [26], Case Management Process (CMP) are "driven by emergent knowledge about the case subject or the environment; largely based on human expertise; highly unpredictable; difficult to replicate; hard to analyze and improve as no HOWTOs available" [26]. Cases differs slightly from KIP because of the point of view adopted. Within the common points, we can find the unpredictability and nonrepeatability (KIP allows each process instance to differs, exactly like each case is unique) from which we can deduce the low or nonexistent structure, and also the knowledge-intensive property where knowledge workers (KIP) or case managers (ACM) must deal with a lot of information and experience to take a decision. A mapping between KIP characteristics from [27] and multiple definitions of case management has been made in [25]. Major distinctions come from the goaloriented property (each case/instance must have one or more goals that evolve with time) and data-centered property (cases are updated with information given by each service or human consulted). [28] also adds boundaries (context and scope of the case) and event-driven (activities are executed following events).

Beyond ACM, multiple case-oriented applications were and are investigated. As mentioned in [25], case handling initialized multiple approaches with the case idea: "Case Management, Adaptive Case Management, Dynamic Case Management, Production Case Management, and Emerging Case Management". As another example, Case-Based Reasoning (CBR) [29] also tries to “... solve(s) problems by relating some similar previously solved problems (past experiences) stored in a case base ..." [29] by using artificial intelligence. CBR is using a problem description to propose solutions.

\section{Motivation, Problems, And Solutions FOR KNOWLEDGE INTENSIVE PROCESSES}

We gather papers in three main steps. First, we search for the terms "weakly structured" "knowledge intensive process" models qualities in google scholar and relationships "weakly structured" "knowledge models" taxonomy. The extracted articles are kept if they are focusing on KIP or KM in their title and abstract. Second, we searched within AdaptiveCM proceedings of 2012, 2015, 2016, and 2018 in order to extract ACM works proposing practical solutions. The case point of view is preferred because some results in the first step are directly coming from the ACM community or are adopting its point of view, and we chose this point of view for the proposed solution. Finally, extra works from previous researches about modeling languages for KIP are added. Some KIP papers may be missing as the goal of the research evolves from works talking about processes' without structure or weakly one, to KIP and their challenges. We organize these papers in three groups:

The first group includes the solutions grounded on BPM practices. These works extend BPM in order to support KIP and to provide an appropriate level of flexibility and user support for KIP management.

In the second group, we discuss the works that exploit new paradigms for KIP management, study characteristics and propose theoretical foundations for KIP. In these works, KIP management is considered as a discipline that is an alternative to (and not an extension of) BPM.

The third group of works reflects the pragmatic views on KIP. Here we discuss the works focusing on the practitioners' needs and presenting concrete solutions for KIP execution support. This group is specifically oriented on already implemented solutions (as opposed to theories and characteristics from the second group) which are not derived from BPM (as opposed to the first group).

\section{A. Extension of BPM for KIP}

KIP or processes that strongly rely on human decisions, collaboration or knowledge always existed in the organizations. BPM develop a theory and practices to help organizations in optimizing their processes [2][6][14]. The rapid expansion of BPM and process automation, proliferation of BPMN as a de-facto standard for process modeling in the beginning of 2000s revealed that KIP can have only limited support from BPM. Van Elst et al. [30] study how KIP can be supported by process models and workflow. Authors emphasize that KIP are exploratory, non-repetitive in detail, and not completely known à priori. Authors conclude that by nature, KIP can't be supported by traditional BPM, yet some BPM practices might improve quality and efficiency of knowledge work, and even improve the process modeling activity itself. Herrmann et al. [31] also recognize flexibility in KIP as a crucial factor for their efficiency. As traditional BPMS and WfMS require predefined structures to optimize business productivity, they are not suitable for KIP management.

Various KIP-supporting solutions based on BPM extensions and adaptations are proposed during the last decade:

Van der Aalst et al. [13] propose a declarative specification language for workflows. They use constraints to define the space of process execution scenarios along with activities and events. A declarative specification simplifies the model as less scenarios need to be modeled. It also leaves more flexibility to the process executor as some activities can be chosen at run-time. 
BPMN 2.0 [11] replaces its predecessor in 2011 and defines several KIP-friendly concepts. One of them is an ad-hoc subprocess, which allows modeler to define a group of activities without sequences between them. Other features enable more flexible scenario execution: one can specify a sub-process where activities will be executed as many time as the process executor requires. New notation also allows for explicit dependencies between activities and data.

Riss et al. [32] study how PAIS are deployed to increase productivity at the cost of rigidity. They propose an approach that keeps flexibility and control with a bottom-up process development and evolution. This approach uses knowledge workers' execution experience in order to make the company's BPM evolving. However, they also state that this approach is based on static models, reducing the ability to adapt to quick changes happening in the real processes or in the regulations.

Shkundina et al. [33] study the challenges a user meets during the knowledge-intensive process execution. The authors conclude that a process workflow provides no context for a concrete task that needs to be executed. As a result, a user spends a lot of time searching for relevant information or guidance in order to make her decisions. The authors propose to enhance the workflow tasks with the information about their context. Some metrics for measuring similarity between tasks based on their execution contexts are proposed.

Moura et al. [34] state that computing infrastructure is not adequate enough to support user activities within KIP. Collaboration support represents a particular challenge. They propose an approach and a tool that recommend during design time and execute at run-time the best dedicated communication services for the KIP.

Manfreda et al. [35] perform a case study to find which BPM methods can be reused in the case of KIP. Globally, the main BPM phases are compatible with KIP, but, local adaptations are required to support innovation and collaboration between the great number of participants. They propose to introduce methods dedicated to KIP into BPM phases, and workshops for initial and modelling existing processes phases. For analysis phase, qualitative methods are preferred. In their specific case study, to-be models are not required.

Sallos et al. [36] linked BPM literature with knowledge intensive entrepreneurship (KIE) literature, in order to build a conceptual framework for business process improvement (BPI) in the context of KIE.

Gromoff et al. [37] outline multiple problems around KIP management in a survey made on 98 russian companies. The problems concerned the process transparency, missing links between KIP events from one instance to another one, few knowledge-intensive control making reporting inefficient, absence of knowledge-intensive change management reducing the predictability, and no system approach hindering the support from the other parts of the organizations. All of these problems motivated the authors to support flexibility for process management in knowledge-intensive perspective. However, authors do not support the idea that a better process formalization will lead to better execution. They propose to improve management of KIP with a self-adaptive system. The system respects KIP's requirements for flexibility within the ideas of process optimization methods and BPMS.

Another addition to BPM is Business Rules Management (BRM) and business rules management systems (BRMS). Following [38], "rules are explicit constraints on behavior and/or provide support to behavior". Moreover, they are explicitly declarative instead of procedural, applied for business (and not for IT), and must be kept within their own repositories in order to be "applied across processes and procedures" [38]. One objective of the rules is to separate the acceptable business activities from unacceptable ones. BRMS are the repositories for storing and integrating business rules within organizations in order to facilitate business processes [39].

Whereas a significant effort is made to extend the existing BPM theory and practices in order to support KIP, other works consider that KIP-supporting solutions do not necessarily have to root in BPM or its adaptation.

\section{B. Theoretical Foundations for KIP}

In this group, we discuss the works that study KIP characteristics and create the foundations for future artifacts and KIP-supporting solutions.

Işik et al. in [14] study the differences between $K I B P$ (KIP) and non-KIBP (regular processes). They list the most used or exposed characteristics found in the literature, in order to test which criteria are unique to KIP. Three criteria discriminates the two kinds of processes: KIP usually are complex, non- or few repeatable, and require creativity ; regular processes usually are simple, repeatable, and do not require a lot of creativity. However, following their study, structure and automation were not discriminating. Precisely, they stated that some KIP, or some parts of them, might be automated.

Kushnareva et al. [40] study limitations of BPMN in the context of a specific case of crisis management process where decisions cannot lead to predefined activities because of the unpredictability of events. The proposed solution is using the statecharts formalism [41] in order to change the point of view (from the "HOW it must be done" to "WHAT must be done" [40]). This work is formalized [26] by using higraphs [42] (mathematical graphs).

Hull et al. [43] introduce the Guard-Stage-Milestone (GSM) meta-model (which is also called GSM-ArtiFact) for lifecycle models. This approach is considered as entity-centric and is based on four key constructs: information model (data, events, and milestones concerning the stage), guard (required events to enter into a stage), stage bodies (container of sub-stages also composed of guards and milestones), and milestones (business goal(s) which ends the current stage instance when activated). The execution evolves in response to the events triggered by milestones and detected by guards.

Dos Santos et al. [44] emphasize that KIP contain creative tasks, tacit knowledge, unpredictable decisions, and evolves dynamically following all of those characteristics. The knowledge manipulation, and how it is still difficult to represent in 
the process models, is a specific aspect that hinders KIP modeling with traditional methods. Specific knowledge-oriented techniques are able to describe some aspects of KIP, but not all of these aspects simultaneously. In order to handle all of the useful concepts, KIPO (Knowledge-Intensive Process Ontology) has been built following the 101 Methodology [45]. KIPO is an high level ontology based on 5 pre-existing ontologies: Business Process Ontology (BPO), Collaborative Ontology (CO), Decision Ontology (DO), Business Rules Ontology (BRO), Knowledge-Intensive Process Core Ontology (KIPCO). Each pre-existing ontology describes a specific point of view, based on criteria gathered from a literature review. KIPO is domain agnostic and its concepts are enriched with stereotypes from Unified Foundational Ontology [46], an ontology built using philosophical, linguistics, and cognitive psychology theories. All of these concepts allow to represent nearly any part of a KIP.

Di Ciccio et al. [27] note that following the research trend there is no holistic system supporting knowledge workers and process. Therefore, they acknowledge a missing mapping between characteristics and system requirements in KIP. The mapping would ease the choice of the existing approaches for practitioners and researchers following the point of view used on KIP. The authors gathered 25 requirements induced by 8 characteristics of KIP. The characteristics were derived from a literature review and 3 application scenarios. Following these characteristics, KIP can be described by: knowledge-driven, collaboration-oriented, unpredictable, emergent, goal-oriented, event-driven, constraint- and rule- driven, non-repeatable. The requirements are organized within 7 classes: data, knowledge actions, rules and constraints, goals, processes, knowledge workers, environment. Following authors, these requirements and characteristics allow to rethink the process life cycle. Instead of the regular design, execute, monitor, analyze, and re-design steps, it becomes easier to think about templates and fragments to reuse in a design, execution, and adaptation continuum.

Marin et al. [25] reuse Di Ciccio et al. [27] KIP characteristics and requirements in order to compare them with current case management definition and test how well CMMN can implement KIP. Case Management definitions mainly support 7 of 8 characteristics (one of them is slightly supported by one definition). The characteristics of KIP were classified between 2 categories : modelling (4) and execution (4). CMMN supports all of the 4 modelling characteristics of KIP ( 2 fully, 2 partly), making it a good candidate for KIP modeling.

Zensen et al. [47] compare BPMN and CMMN using an example of KIP and provide guidelines for their usage. They study four characteristics of each modeling language: process structure, routing and control-flow, communications and events, data aspect and data-flow. Whereas BPMN is perfect for repetitive work with few data exchange, few exceptions, and strict workflow, CMMN is data-centered and allows for more flexibility in ordering tasks within a workflow. Their findings show that the two languages are complementary: Each modeling language can potentially integrate the other for a specific activity or task.

Hislop et al. [48] define a gap between theory and practice in KM field. They propose directions for future research in KM field, based on multiple papers. According to the authors, $\mathrm{KM}$ can benefit from IT in order to increase socialising, connectivity and collaboration in order to create and co-create knowledge. Multiple technologies are presented as examples. Nevertheless, the biggest organizations, or the most modern ones, are still unable to benefit from those technologies. Too much information is available within organizations, but multiple factors (technical and organizational) hinder the possibilities of their usage by knowledge workers.

All of these characteristics specific to KIP introduce new solutions, which represent alternatives to BPM and workflowinspired approaches.

\section{Execution Support for KIP}

Di Ciccio et al. [22] study the informal communications and processes generated on the fly in certain situations. They propose MailofMine, a tool that builds workflow structure from a collection of mails, in order to represent the artful processes (KIP) implicitly hidden behind the communications. Based on process mining or workflow mining techniques, it extracts tasks and presents them graphically with a global and a local views. On local view, each task is shown with its constraints: time (before/after another task), implication (required tasks), repeatability (how many times each may be done). In global view, all the tasks are presented with their interdependencies or constraints (orders between tasks, and how many times they may be achieved). The difference between local and global view lies on the readability criteria. The local view is more adapted to check the constraints of one task, and the global view is more adapted to have a main view of the whole process. KIP are therefore considered as a collection of constraints.

Moura et al. [34][49] aim to support the execution of knowledge intensive activities requiring collaboration. KIP usually require collaboration between multiple actors and services, but authors argue that infrastructures are not able to support these activities. They propose XcuteKIP, a semi-automatic recommendation system of collaborative services for KIP, based on the previously shown KIPO [44] (precisely by using the Collaborative Ontology). Each collaborative activity of a KIP is mapped to services, in order to calculate the most appropriate collaborative service to run the process and make people communicate adequately.

In addition to these original solutions, ACM appeared as a new domain of research. Some particularities of ACM make it interesting:

- ACM manipulates cases, which can be view as an equivalent of a process instance (each instance/case is somewhat different to another one, following events). These cases can be stored when finished, and reused via templates [50] to help knowledge worker make better decisions. 
- ACM uses goals to help knowledge worker to choose the best action to do. Each case might have multiple goals or sub-goals, offering multiple choices for each step.

- ACM is data-centric. Because of unpredictability, instead of using the activity-centered view where the instance is moving from one state to another one on a predefined workflow model following rules, each case can be seen as a file where data are added or modified by knowledge workers and/or resources which generate data.

The ACM community studied multiple characteristics of this point of view, and proposed some solutions to handle cases within information systems.

Huber et al. [24] study ACM in order to fill the gap left between BPM and KIP. BPM is typically used to increase the performance of processes by standardizing repetitive work with WfMS. As KIP are unstructured by nature and require flexibility, BPM is unable to correctly support them and increase their efficiency. However, ACM is proposed to fill the gap by giving a general structure from previous cases, and leave the exact execution open to change. The main problem with this idea, is how to apply ACM in practice within organizations. Some questions around scaling in large companies and how implied collaboration would work are opened.

Huber et al. [51] study ACM in the context of Open Innovation. Companies are creating more complex products and services, requiring cooperation between multiple teams. Open Innovation (OI), a paradigm supporting collaboration, includes creative activities that are unpredictable and highly knowledge-intensive. The authors try to use ACM to support these unpredictable and knowledge-intensive activities which requires a lot of collaboration.

Cognini et al. [19] study how to partially structure processes depending on the possible variances. As knowledge work is not predictable, there usually are no predefined structure available to help a knowledge worker to take a decision. Authors are using CBR methodology in order to use process fragments and propose solutions to knowledge workers. They propose a "case-based process fragment modelling language named Business Process Feature Model (BPFM)", which can be used as a "case content representation (modelling language)". BPFM is compared to BPMN, CMMN, and DECLARE [52] modelling languages to expose the required features supported or missing. As CBR is used in the execution context, BPFM is used to follow the steps/activities realized and the data used.

Tran et al. [53] propose an approach to allow flexibility within processes while checking its compliance, using logic rules in ACM. Therefore, ISIS Papyrus ACM System [54] is extended to support compliance checking. During design time, the usual methods of model checking can be applied. However, at run-time, as goals evolve, state-based rules and data-based rules are used to propose a predefined set of possible goals and tasks. New tasks can be proposed from generic templates, in this case, the knowledge worker takes the responsibility of complying with the rules. When tasks are completed, their results are gathered to create new compliance rules to achieve goals.

Tenschert et al. [55] present an approach to detect micro processes by using speech acts. Instead of monitoring actions, the progress of knowledge work is based on interactions. Authors expect to "bridge the gap between structured, semistructured and ad-hoc processes" by focusing on interactions. The approach allows to create templates that reduces the need to seek information within documents, and help knowledge worker to automatically fill (partially or fully) data depending on the current case.

Czepa et al. [56] discuss how to ease the update of compliance rules for knowledge workers and business users using constraints and ontologies. Machine learning techniques are used to learn from past cases, and recommend next actions.

Routis et al. [57] propose directions to upgrade CMMNenabled platforms. Authors stated limitations within the platforms. Typically, conditions on data or event are not supported, closed cases are not well exploited when reused, knowledge workers can't make modification on case model during execution phase. The notion of Case Learning is introduced as the procedure to improve case model when the context changes by offering suggestions instead of restrictions from the CMMN flow. A first goal would be to implement a method that could transform a CMMN model into a Case Template, and vice versa. The second goal is to validate the Case Learning procedure as a useful tool for knowledge workers.

Bider et al. [58] test if patterns could be used in nonworkflow models, precisely with goals patterns within STateoriented Business Process Modeling (SToBPM). SToBPM represents the state of a process instance within a dimensional space as a vector. Multiple types of data can be embedded as parameters of the process instance. Patterns used in this approach have characteristics: first, they are not activities/tasks patterns with their order like in workflow models ; second, "the patterns are rather semantic than syntactic". This early test shown interesting positive results, even if it was very specific to SToBPM and goals patterns.

Zasada [59] proposes to derive patterns from legal texts, using content analysis methods, in order to help elicitation of food industry compliance requirements. Patterns are extracted from legal texts using content analysis in a semi-automated procedure, and are stored as regular expressions. Even if the method is still not fully automated, the generated rules are a machine readable knowledge that can be reused.

Sid et al. [60] focus on flexibility in KIP by using artificial intelligence planning techniques. They allow knowledge workers choose any granularity level during execution, and dynamically change goals. Their solution aims to support the knowledge worker in achieving its goals instead of giving him too much freedom with few control or excessive restrictions on the given recommendations.

\section{DISCUSSION}

In spite of the recent advancements, KIP management remains challenging for practitioners and many research ques- 
tions are yet to be solved by researchers. In this section we summaries the challenges and outline some perspectives of the future research in KIP.

The reported literature acknowledges various aspects of KIP that make them different from regular business processes and, thus, challenging to automate and manage with already established methods and tools [32][34].

Based on our study, the following questions still need to be addressed by researchers and practitioners in the future. We illustrate these questions with an example of a meeting deciding on the orientation to take for a company. A meeting can be seen as a KIP as it implies a lot of collaboration between multiple participants, creativity is required to bring new solutions, decisions are often made, and each meeting is unique.

A. How to manage efficiently and effectively the information and knowledge that is manipulated (i.e., created, used, updated) by KIP?

According to [48], many organizations struggle with the amount of information they have to manage. Lack of information and knowledge management practices and tools, and lack of integration between knowledge management and process management have a negative impact on KIP.

Whereas ACM proposes some solutions that address knowledge-intensity "by design" [51], BPM solutions require an extension [35].

In our example, recording each participants' speeches and documents used would help to see how a decision has been made in the case where the team has to change. However, the huge amount of texts, documents, and meetings are hindering their usage: few people have time to hear or read again everything. One solution is to extend documents and transcriptions by adding relevant tags on them (which document has been used to take which decision). Another solution would be to follow Adaptive Open Innovation [51] phases instead.

\section{B. How to support collaboration and context-specific decision making for KIP?}

Collaboration between knowledge workers participating in KIP remain challenging [34]. According to [32], the organizations are often preoccupied with process standardization and control. Supporting collaboration and creativity is not a priority for them. An excess of control or formalization has a negative impact on knowledge workers and KIP [37]. [22] offers help for informal communications, but solutions for collaboration support in highly-controlled environments are yet to be developed.

In the illustrative example, meetings are usually made around a table using pencils and papers, and optionally with video-conference for distant people. Forcing people to use a precise software instead of their own tools might reduce their creativity and freedom, therefore breaking the goal of the meeting: presenting and sharing creative ideas in order to take decision. As there are no known solutions to combine standardization and creativity, only homemade adaptations may work.

\section{How to integrate context information in KIP design?}

A perspective to design and build a KIP supporting system based on some "à priori" model (by analogy with BPMS and WfMS) is attractive: model simulation, validation and verification enable compliance "by design" and could save a lot of efforts in implementation. KIP however are contextor case-sensitive: each of their execution scenario is unique. Compared to workflow execution, KIP execution can be considered "unpredictable" as its execution scenario is driven (a) by a context (a current situation) and (b) by a tacit knowledge of an expert (or group of experts) involved.

A traditional workflow model does not provide enough flexibility to support KIP. Within current solutions, the workflow can be partially skipped or organised as flexible ad-hoc subprocess [11], however a new activity cannot be added to the workflow at run-time if not planned in advance.

Declarative modeling languages propose a partial solution: if the activity does not violate predefined constraints, it can be executed [53].

In our example, each meeting has precise objectives. Even if the main phases are globally known (agenda's presentation, and discussion of each topic), the knowledge brought by each person might change the agenda based on the internal and external context of the company. Foreseeing these information while building the agenda is difficult as the situation evolves continuously based on the talks and decisions. Constraints, like prioritization and time limits, avoid unending talks on unexpected and useless topics. Sharing the agenda and main information before the meeting also reduce off topic talks.

\section{How to support compliance in KIP?}

Frequent changes in regulations and rules have to be followed by the processes and have to be reflected by corresponding PAIS [56][61]. BRM brought solutions for compliance by managing rules within specific transverse repositories using dedicated languages [62]. Rules and declarative approaches allow to create a frame where knowledge workers are free as long as they stay inside. For KIP, automated compliance checking is a challenge: due to context-sensitivity, compliance checking in KIP needs to be done at run-time, when some activity is created and/or instantiated according to the execution context. Decisions about the next activity to execute are often made by a knowledge worker in KIP [40]. Thus, it is the responsibility of a knowledge worker to ensure that this activity comply with the regulations and norms. A possibility to analyze an activity instance, its compliance and its potential impact on the process scenario would be of a great value for knowledge workers [53][56].

The lack of formalization in regulations and norms also makes compliance checking in KIP challenging. These regulations and norms are often specified as an unstructured text, which is hard to access and to use for knowledge workers during the process execution [61]. Formalizing regulations and integrating them into PAIS can improve the situation and help the knowledge worker to ensure the compliance [59]. 
In the example, the order of the agenda might be very important. It is the responsibility of each participant to present relevant ideas. However, it is difficult to prevent the discussions to move on another topic, except if the agenda is regularly reminded. In some emergency cases, it might be required to change of topics.

\section{E. How to support flexibility in KIP scenario?}

Flexibility in KIP is studied in multiple works [32][31][61][37][53][51][47]. Some works tried to offer some solutions for managing flexibility in BPM, like [11]. However, the paradox of organizations expecting to standardize their processes with BPM, and respecting regulations, while giving enough freedom of action to their knowledge worker is still unsolved. KIP are overlapping multiple fields of research, like BPM and KM, allowing multiple points of views. In the illustrative example, flexibility is available by the nature of a meeting: people are talking following simple rules (people raise their hands before having their speaking slot). If everybody is in accordance to, an unexpected topic can be discussed even if not on the agenda. However, if no agreement is found, it becomes difficult to add a topic.

Two points of view on KIP are prevalent today: an activitycentered view where the process is specified with a sequence of activities, and a data-centered view, where the process is specified with the data (also referred to as a "case") it requires or manipulates.

Using the activity-centered view, the process designer can explore the process (KIP) execution logs in order to analyze the process steps and identify the process structure [22]. The activity-centered view highlights structure, and focuses on the order of activities. Here, some high level structures [24] or patterns [19] can be explored.

Within the data-centered view, a KIP seeks to achieve one or multiple goals through collaboration between participants. KIP can be managed using a document (a case folder). Each participant uses an information from the case folder and makes decisions based on it. Participants can execute activities for the case based on the available inputs and required outputs [2]. This point of view ensures more flexibility in decisions and process scenarios compared to workflow (or activity-centered view).

The two points of view are complimentary: the activitycentered view can be used to provide a global "roadmap" for a KIP - a sequence of steps to achieve the goal(s) - at design time. This roadmap is not hindering local flexibility for complex steps. These steps (or subprocesses) can be detailed using the data-centered view at run time. Conversely, patterns for some complex (or highly prescriptive) activities can be defined at design time, using the activity-centered paradigm. At run-time, a knowledge worker can choose a pattern or a combination of patterns when appropriate, maintaining the global flexibility.

Multiple ideas of patterns applied to KIP or ACM are discussed. In [59], compliance patterns are studied within legal texts and transformed into regular expressions in order to check compliance. [58] introduces goal patterns for SToBPM. [19] represented cases with CBR and BPFM in order to help knowledge workers by proposing solutions based on process fragments.

Using activity-centered point of view on the illustrative example would give a list of topics to talk about. In the strict vision, it would be impossible to talk about something else than the current topic. As a meeting is by nature flexible, applying the data-centered point of view allows to change of topic following the information given by each participant. Some topics might be linked because of their impact on the company, they must therefore be addressed together and in a precise order. In this case, the linked topics added in the agenda can be seen as fragments of patterns.

\section{F. How to explore and reuse process fragments in KIP?}

The idea of studying past cases in order to identify and reuse fitting fragments in the next instances (like in CBR) of KIP is highlighted in [27][19] and seems to have a great potential. A possible way to help knowledge workers could be to provide patterns of past solutions, and check compliance of an instance compared to a reference, by using a combination of mathematical technique and ontologies. We expect to compare the logs of activities of current instance of KIP, with the previous finished KIP or reference from regulations and standards, similarly to what CBR does when searching the most close solution to a problem.

The illustrative example is based on an agenda and topics. In order to build the next agenda on specific topics already addressed in the past, it might be useful to reuse past fragments. If these topics were not added in the initial agenda, but during the unexpected deviation of talks, it might be useful to reuse this order in the new agenda as a reminder of the context to every participant.

In [9], several methods are combined to evaluate the similarity of business process models. Similarly, three techniques can be combined in order to check the structure, the semantic, and the behavior of process fragments. In order to compare the structure of patterns, we propose to use Formal Concept Analysis (FCA) [63]. FCA is a method for data structuring that is based on deriving a concept hierarchy from a collection of objects and their properties [64][65]. This method is applied for knowledge discovery in databases (KDD) [66]. The semantic comparison is expected to be done using concepts from BabelNet [67] or semantic distance from WordNet [68]. Behavior comparison can be done using association rules, like A Priori [69]. Association rules generate rules describing in which conditions some objects might appear, based on traces of execution.

We expect to show first how FCA and semantic analysis can generate interesting fragments, then how association rules can propose an order of assembly. The first contribution will extract fragments of interest for the business based on its domain ontology. The second contribution will propose to the knowledge worker how to combine these fragments. 


\section{CONCLUSION}

Knowledge-intensive process management represents a challenge for modern organizations: KIP are often complex and context-specific; they require flexibility; they involve collaboration and rely on creativity and tacit knowledge of participants. Product design, change management, problem solving, diagnostics, customer relationship management, education, apprenticeship - are some examples of KIP.

Strategic modeling activities share common practices and challenges with KIP: first priority on goals; information processing, collaboration, decision making as usual steps; importance of compliance or constraint while executing a process. Contributions from the KIP domain bring answers and practices to strategic modeling.

In this article we provided an overview of the current work on KIP. We examined several groups of contributions:

- the works defining KIP management solutions as an extension of BPM solutions. These works use the activitycentered point of view on KIP and add new concepts and semantics in order to increase the run-time flexibility of processes and improve the context-awareness.

- the works developing theoretical foundations for KIP and considering KIP management as an alternative to BPM. These works explore alternative, non-workflow approaches to process management (data-centered, artifactcentered, etc).

- the works proposing pragmatic solutions for KIP execution support. These works are driven by the practitioners needs and focused on implementation and practical value on the first place.

In spite of significant efforts of researchers and practitioners in the domain, more research is still required in order to enhance PAIS with KIP management capabilities: some major challenges concern run-time flexibility, standardization and compliance support. Run-time flexibility refers to a capacity of a PAIS to add, skip or modify process tasks at run-time. Standardization and compliance support refers to a capacity of a PAIS to help knowledge workers to respect regulations. Context-specific guidance for knowledge workers, automatic identification of compliant and non-compliant activities at runtime are possible solutions.

Patterns in KIP and process execution context are studied in the literature. Identifying patterns in previous activities and resolved cases/instances can be helpful for developing recommendations for knowledge workers. For example, a corresponding solution can help a knowledge worker to identify an activity that fits the situation and, at the same time, comply with the norms and regulations defined for current KIP instance. We are going to develop this idea in the future work.

\section{REFERENCES}

[1] M. Von Rosing, H. Von Scheel, and A.-W. Scheer, The Complete Business Process Handbook: Body of Knowledge from Process Modeling to BPM. Morgan Kaufmann, 2014, vol. 1, "Phase 1: Process Concept Evolution".
[2] K. D. Swenson, N. Palmer et al., Mastering the unpredictable: how adaptive case management will revolutionize the way that knowledge workers get things done. Meghan-Kiffer Press Tampa, 2010, vol. 1.

[3] OMG, Case Management Model and Notation, Version 1.1, Object Management Group Std., Rev. 1.0, Jan. 2016. [Online]. Available: http://www.omg.org/spec/CMMN/1.1

[4] M. Marin, R. Hull, and R. Vaculín, "Data centric bpm and the emerging case management standard: A short survey," in International Conference on Business Process Management. Springer, 2012, pp. 24-30.

[5] P. H. P. Richetti, J. C. de AR Gonçalves, F. A. Baião, and F. M. Santoro, "Analysis of knowledge-intensive processes focused on the communication perspective," in International Conference on Business Process Management. Springer, 2017, pp. 269-285.

[6] M. Dumas, M. La Rosa, J. Mendling, H. A. Reijers et al., Fundamentals of business process management. Springer, 2013, vol. 1.

[7] W. Gish, "Definition of strategy models," https://smallbusiness.chron com/definition-strategy-models-15431.html, [Online; accessed July2019].

[8] M. Ghasemi and D. Amyot, "From event logs to goals: a systematic literature review of goal-oriented process mining," Requirements Engineering, pp. 1-27, 2019.

[9] A. Schoknecht, T. Thaler, P. Fettke, A. Oberweis, and R. Laue, "Similarity of business process models-a state-of-the-art analysis," ACM Computing Surveys (CSUR), vol. 50, no. 4, p. 52, 2017.

[10] M. Weske, Business Process Management: Concepts, Languages, Architectures. Springer, 2007.

[11] OMG, Business Process Model and Notation (BPMN), Version 2.0, Object Management Group Std., Rev. 2.0, January 2011. [Online]. Available: http://www.omg.org/spec/BPMN/2.0

[12] G. Keller, A.-W. Scheer, and M. Nüttgens, Semantische Prozeßmodellierung auf der Grundlage” Ereignisgesteuerter Prozeßketten (EPK)”. Inst. für Wirtschaftsinformatik, 1992.

[13] W. M. van Der Aalst, M. Pesic, and H. Schonenberg, "Declarative workflows: Balancing between flexibility and support," Computer ScienceResearch and Development, vol. 23, no. 2, pp. 99-113, 2009.

[14] Ö. Işik, W. Mertens, and J. Van den Bergh, "Practices of knowledge intensive process management: quantitative insights," Business Process Management Journal, vol. 19, no. 3, pp. 515-534, 2013.

[15] A. Abecker, A. Bernardi, K. Hinkelmann, O. Ku, M. Sintek et al., "Context-aware, proactive delivery of task-specific information: The knowmore project," Information Systems Frontiers, vol. 2, no. 3-4, pp. 253-276, 2000.

[16] J. B. França, F. A. Baião, and F. M. Santoro, "Towards characterizing knowledge intensive processes," in Computer Supported Cooperative Work in Design (CSCWD), 2012 IEEE 16th International Conference on. IEEE, 2012, pp. 497-504.

[17] M. B. Hassen, M. Turki, and F. Gargouri, "Choosing a sensitive business process modeling formalism for knowledge identification," Procedia Computer Science, vol. 100, pp. 1002-1015, 2016.

[18] J. Syed, P. A. Murray, D. Hislop, and Y. Mouzughi, The Palgrave handbook of knowledge management. Springer, 2018.

[19] R. Cognini, K. Hinkelmann, and A. Martin, "A case modelling language for process variant management in case-based reasoning," in International Conference on Business Process Management. Springer, 2016, pp. 30-42.

[20] Z. Panian, "A promising approach to supporting knowledge-intensive business processes: business case management," World Academy of Science, Engineering and Technology, vol. 75, pp. 642-648, 2011.

[21] C. Hill, R. Yates, C. Jones, and S. L. Kogan, "Beyond predictable workflows: Enhancing productivity in artful business processes," IBM Systems journal, vol. 45, no. 4, pp. 663-682, 2006.

[22] C. Di Ciccio, M. Mecella, M. Scannapieco, D. Zardetto, and T. Catarci, "Mailofmine-analyzing mail messages for mining artful collaborative processes," in International Symposium on Data-Driven Process Discovery and Analysis. Springer, 2011, pp. 55-81.

[23] W. M. Van der Aalst, M. Weske, and D. Grünbauer, "Case handling: a new paradigm for business process support," Data \& Knowledge Engineering, vol. 53, no. 2, pp. 129-162, 2005.

[24] S. Huber, M. Lederer, and F. Bodendorf, "It-enabled collaborative case management-principles and tools," in Collaboration Technologies and Systems (CTS), 2014 International Conference on. IEEE, 2014, pp. 259-266.

[25] M. A. Marin, M. Hauder, and F. Matthes, "Case management: an evaluation of existing approaches for knowledge-intensive processes," in 
International Conference on Business Process Management. Springer, 2016, pp. 5-16.

[26] E. Kushnareva, I. Rychkova, and B. Le Grand, "Semantics of higraphs for process modeling and analysis," in 2016 IEEE 20th International Enterprise Distributed Object Computing Workshop (EDOCW). IEEE, 2016, pp. $1-8$.

[27] C. Di Ciccio, A. Marrella, and A. Russo, "Knowledge-intensive processes: characteristics, requirements and analysis of contemporary approaches," Journal on Data Semantics, vol. 4, no. 1, pp. 29-57, 2015.

[28] I. Rychkova, B. Le Grand, and C. Souveyet, "Towards executable specifications for case management processes," in Advances in Intelligent Process-Aware Information Systems. Springer, 2017, pp. 49-77.

[29] S. K. Biswas, N. Sinha, and B. Purkayastha, "A review on fundamentals of case-based reasoning and its recent application in different domains," International Journal of Advanced Intelligence Paradigms, vol. 6, no. 3, pp. 235-254, 2014.

[30] L. Van Elst, F.-R. Aschoff, A. Bernardi, and S. Schwarz, "Weaklystructured workflows for knowledge-intensive tasks: An experimental evaluation," in Enabling Technologies: Infrastructure for Collaborative Enterprises, 2003. WET ICE 2003. Proceedings. Twelfth IEEE International Workshops on. IEEE, 2003, pp. 340-345.

[31] C. Herrmann and M. Kurz, "Adaptive case management: supporting knowledge intensive processes with it systems," in International Conference on Subject-Oriented Business Process Management. Springer, 2011, pp. 80-97.

[32] U. V. Riss, A. Rickayzen, H. Maus, and W. M. van der Aalst, "Challenges for business process and task management," Journal of Universal Knowledge Management, vol. 2, pp. 77-100, 2005.

[33] R. Shkundina and S. Schwarz, "A similarity measure for task contexts." in ICCBR Workshops, 2005, pp. 261-270.

[34] E. V. Moura, F. M. Santoro, and F. A. Baião, "Collaboration support for knowledge-intensive processes through a service-based approach," in Computer Supported Cooperative Work in Design (CSCWD), 2013 IEEE 17th International Conference on. IEEE, 2013, pp. 319-324.

[35] A. Manfreda, B. Buh, and M. Indihar Štemberger, "Knowledge-intensive process management: a case study from the public sector," Baltic Journal of Management, vol. 10, no. 4, pp. 456-477, 2015.

[36] M. P. Sallos, E. Yoruk, and A. García-Pérez, "A business process improvement framework for knowledge-intensive entrepreneurial ventures," The Journal of Technology Transfer, vol. 42, no. 2, pp. 354-373, 2017.

[37] A. Gromoff, Y. Bilinkis, and N. Kazantsev, "Business architecture flexibility as a result of knowledge-intensive process management," Global Journal of Flexible Systems Management, vol. 18, no. 1, pp. 73-86, 2017.

[38] R. G. Ross, "The business rules manifesto," http://www. businessrulesgroup.org/brmanifesto.htm, 2003, [Online; accessed May-2019].

[39] I. Graham, Business rules management and service oriented architecture: a pattern language. John wiley \& sons, 2007.

[40] E. Kushnareva, I. Rychkova, and B. Le Grand, "Modeling and animation of crisis management process with statecharts," in International Conference on Business Informatics Research. Springer, 2015, pp. 145-160.

[41] D. Harel, "Statecharts: A visual formalism for complex systems," Science of computer programming, vol. 8, no. 3, pp. 231-274, 1987.

[42] —_, "On visual formalisms," Сommun. АCM, vol. 31, no. 5, pp. 514530, 1988

[43] R. Hull, E. Damaggio, F. Fournier, M. Gupta, F. T. Heath, S. Hobson, M. Linehan, S. Maradugu, A. Nigam, P. Sukaviriya et al., "Introducing the guard-stage-milestone approach for specifying business entity lifecycles," in International Workshop on Web Services and Formal Methods. Springer, 2010, pp. 1-24.

[44] J. B. dos Santos França, J. M. Netto, J. do ES Carvalho, F. M. Santoro, F. A. Baião, and M. Pimentel, "Kipo: the knowledge-intensive process ontology," Software \& Systems Modeling, vol. 14, no. 3, pp. 1127-1157, 2015.

[45] N. F. Noy, D. L. McGuinness et al., "Ontology development 101: A guide to creating your first ontology," 2001.

[46] G. Guizzardi, "Ontological foundations for structural conceptual models," Ph.D. dissertation, University of Twente, 2005.

[47] A. Zensen and J. Küster, "A comparison of flexible bpmn and cmmn in practice: A case study on component release processes," in 2018 IEEE 22nd International Enterprise Distributed Object Computing Conference (EDOC). IEEE, 2018, pp. 105-114.
[48] D. Hislop, P. A. Murray, A. Shrestha, J. Syed, and Y. Mouzughi, "Knowledge management:(potential) future research directions," in The Palgrave Handbook of Knowledge Management. Springer, 2018, pp. 691-703.

[49] E. V. Moura, F. M. Santoro, and F. A. Baião, "Xcutekip: support for knowledge intensive process activities," in CYTED-RITOS International Workshop on Groupware. Springer, 2015, pp. 164-180.

[50] I. Bider, "Towards process improvement for case management," in International Conference on Business Process Management. Springer, 2016, pp. 96-107.

[51] S. Huber, P. Schott, and M. Lederer, "Adaptive open innovation: solution approach and tool support," in Proceedings of the 7th International Conference on Subject-Oriented Business Process Management. ACM, 2015, p. 12.

[52] M. Pesic, H. Schonenberg, and W. M. Van der Aalst, "Declare: Full support for loosely-structured processes," in 11th IEEE International Enterprise Distributed Object Computing Conference (EDOC 2007). IEEE, 2007, pp. 287-287.

[53] T. Tran, E. Weiss, C. Ruhsam, C. Czepa, H. Tran, and U. Zdun, "Embracing process compliance and flexibility through behavioral consistency checking in acm: A repair service management case," in Business Process Management Workshops. BPM 2016., 2015.

[54] "Isis papyrus acm system," https://www.isis-papyrus.com/e15/pages/ business-apps/acm.html, [Online; accessed April-2019].

[55] J. Tenschert and R. Lenz, "Supporting knowledge work by speech-act based templates for micro processes," in International conference on business process management. Springer, 2016, pp. 78-89.

[56] C. Czepa, H. Tran, U. Zdun, T. T. T. Kim, E. Weiss, and C. Ruhsam, "Towards structural consistency checking in adaptive case management," in International Conference on Business Process Management. Springer, 2016, pp. 90-95.

[57] I. Routis, M. Nikolaidou, N. Alexopoulou, and D. Anagnostopoulos, "Empowering knowledge workers with $\mathrm{cmmn}$ : The concept of case learning," in 2018 IEEE 22nd International Enterprise Distributed Object Computing Workshop (EDOCW). IEEE, 2018, pp. 33-36.

[58] I. Bider and G. Koutsopoulos, "Introducing goal patterns for stateoriented business process modeling," in 2018 IEEE 22nd International Enterprise Distributed Object Computing Workshop (EDOCW). IEEE, 2018, pp. 43-47.

[59] A. Zasada, "A box of bricks for modelling domain-specific compliance pattern," in 2018 IEEE 22nd International Enterprise Distributed Object Computing Workshop (EDOCW). IEEE, 2018, pp. 37-42.

[60] I. Sid, M. Reichert, and A. R. Ghomari, "Enabling flexible task compositions, orders and granularities for knowledge-intensive business processes," Enterprise Information Systems, vol. 13, no. 3, pp. 376-423, 2019.

[61] G. De Angelis, A. Pierantonio, A. Polini, B. Re, B. Thönssen, and $\mathrm{R}$. Woitsch, "Modeling for learning in public administrations-the learn pad approach," in Domain-Specific Conceptual Modeling. Springer, 2016, pp. $575-594$.

[62] M. Zur Muehlen, M. Indulska, and G. Kamp, "Business process and business rule modeling languages for compliance management: a representational analysis," in Tutorials, posters, panels and industrial contributions at the 26th international conference on Conceptual modelingVolume 83. Australian Computer Society, Inc., 2007, pp. 127-132.

[63] B. Ganter and R. Wille, Formal concept analysis: mathematical foundations. Springer Science \& Business Media, 2012.

[64] B. Wormuth and P. Becker, "Introduction to formal concept analysis," in 2nd International Conference of Formal Concept Analysis February, vol. 23, 2004

[65] R. Belohlavek, "Introduction to formal concept analysis," Palacky University, Department of Computer Science, Olomouc, vol. 47, 2008.

[66] J. Hereth, G. Stumme, R. Wille, and U. Wille, "Conceptual knowledge discovery and data analysis," in International Conference on Conceptual Structures. Springer, 2000, pp. 421-437.

[67] R. Navigli and S. P. Ponzetto, "Babelnet: The automatic construction, evaluation and application of a wide-coverage multilingual semantic network," Artificial Intelligence, vol. 193, pp. 217-250, 2012.

[68] G. A. Miller, "Wordnet: a lexical database for english," Communications of the ACM, vol. 38, no. 11, pp. 39-41, 1995.

[69] R. Agrawal, R. Srikant et al., "Fast algorithms for mining association rules," in Proc. 20th int. conf. very large data bases, VLDB, vol. 1215, 1994, pp. 487-499. 